\title{
ZONE BASED JOB SCHEDULING IN MOBILE GRID ENVIRONMENT
}

\author{
S. Stephen Vaithiya and S. Mary Saira Bhanu \\ Department of Computer Science \&Engineering, \\ National Institute of Technology, Tiruchirappalli, India \\ stphenvaithiya@gmail.com \& msbenitt.edu
}

\begin{abstract}
Recent advances in mobile computing, and strong interest of the scientific community in the Grid have led to research into the Mobile Grid. One primary issue associated with the efficient and effective utilization of mobile resources in a mobile grid is scheduling of tasks. In this paper, a task scheduling algorithm is proposed based on the dynamic prediction of resource mobility and battery power in the mobile grid environment. The mobility is predicted based on the movement type, movement pattern and movement direction of the mobile resource within the zone. The battery power is predicted based the C-rate of the mobile resource. The availability prediction of a mobile resource is evaluated during the task submission and this allows the system to consider run-time parameters prior to execution. The evaluation study employs a number of experiments with various simulation settings. The simulation results point to the efficacy of the proposed work.
\end{abstract}

\section{KEYWORDS}

Mobile Grid, Scheduling, Movement Model, Movement Type, Movement Direction

\section{INTRODUCTION}

Grid computing is based on the coordinated sharing of distributed and heterogeneous resources to solve large-scale problems in dynamic virtual organizations. With the advent of high bandwidth third-generation mobile networks and other wireless networks, grid computing has migrated from traditional parallel and distributed computing to pervasive and utility computing based on the wireless networks and mobile devices, which results in the emergence of a new computing paradigm named mobile grid. Mobile grid integrates traditional wired grid through wireless channel to share grid resources to mobile users or provide resources to grid [1]. Mobile devices have advantages over fixed computing resources such as mobility, portability, and pervasiveness. These strengths allow mobile grid well-applied to location-restricted fields requiring supportive infrastructure in wildfire prevention, disaster management, and e-health system, etc [2]. The exploding number of mobile devices implies an immense potential of the new breed even if an individual mobile device still has limitations in terms of computing resources and network stability. While integrating grid with mobile devices, the devices can act as service/resource consumer from the grid which offers reliability, performance and quality of service or they can act both as consumer and provider to host grid management services instead of relying on fixed infrastructure. The characteristics of mobile devices such as inferior computing power, low network bandwidth, volatility, battery power, mobility and heterogeneity should be taken into account, to utilize mobile devices as resources. In such a resource-constrained environment, job scheduling plays a crucial role in overall performance. The scheduler should allocate jobs to proper mobile devices, minimize uncertainty in job execution and strive to optimize scheduling objectives such as maximize throughput, response time and balance available resources. 
However, it is impractical to assume that perfect performance information on underlying resources in a mobile grid is readily available. Especially, since the mobile grid is much less stable than the wired environment, dynamism should be considered at the scheduling time. Job scheduling in mobile grids thus require a robust system model that can incorporate all these factors. In order to meet the dynamic and mobile nature of resources, the availability should be predicted.

In [3] we proposed a scheduling algorithm which calculates the Mobility and Battery power availability for scheduling jobs in a single cell. In this paper, it is extended for calculating physical availability both within the cell as well as within zone. Since the zone is considered for predicting the resource provider's physical availability, the residence time of the resource inside the zone increases and this has improved the performance of the proposed system. The physical availability prediction uses the movement type, movement pattern and movement direction of the resource providers. The battery power prediction is based on the C- rate of the battery. The experimental results give the effect of mobility and battery power on execution time when the number of cells in the zone is varied from two to six, and also gives the effect of tasks with varying size on the execution time.

The rest of the paper is structured as follows. Section 2 discusses the related work. Section 3 presents the proposed system model and scheduler for mobile grid. Section 4 describes the proposed scheduling algorithm. Section 5 deals with experimental setup and the performance analysis of the proposed algorithm. Section 6 gives the conclusion of the paper.

\section{RELATED WORK}

Several studies are going on in scheduling issues in mobile grid focusing on power efficiency, communication availability due to mobility, and job replication. Kasula et al. [4] proposes a layered system model to bridge the gap between mobile and grid computing world. The paper addresses the problem of scheduling in the presence of nodes disconnection. Ghosh et al. [5] proposed a game theoretic pricing strategy for efficient job allocation in mobile grids. They proposed a two-player, non-cooperative, alternating-offer bargaining game between the Wireless Access Point Server and the mobile devices to determine a fair pricing strategy, which is then used to effectively allocate jobs to the mobile devices with a goal to maximize the revenue for the grid users. Chang-Qin Huang et al. [6] presents power-aware hierarchical scheduling in wireless Grids. The mobile node selection is targeted to minimize the energy consumed for communication and computation. Farooq et al. [7] devised a generic mobility model to predict a time duration for which a resource will remain in a specific domain. It is based on learning from the resource's behaviour in the past and not by the random movement of mobile devices. JongHyuk Lee et al [8] presents a novel balanced scheduling algorithm in mobile grid, taking into account the mobility and availability in scheduling. This classifies the mobile devices into nine groups based on full availability and partial availability. The availability is calculated by considering the uptime and downtime.

From the above literature it has been observed that either the mobility or battery power of the resource is considered for scheduling tasks in the mobile grid environment. The mobile resources which are considered in this work can act both as consumer and provider. The proposed work quantitatively predicts resource availability dynamically by taking parameters of mobile resources such as the mobility and battery power. The physical availability factor is predicted based on the fixed time period in [3], but in this paper the resource residence time in the zone is considered. Battery availability considers energy consumed for communication and computation of mobile grid user in addition to the mobile user's current usage. 


\section{The Proposed System Model ANd Scheduler}

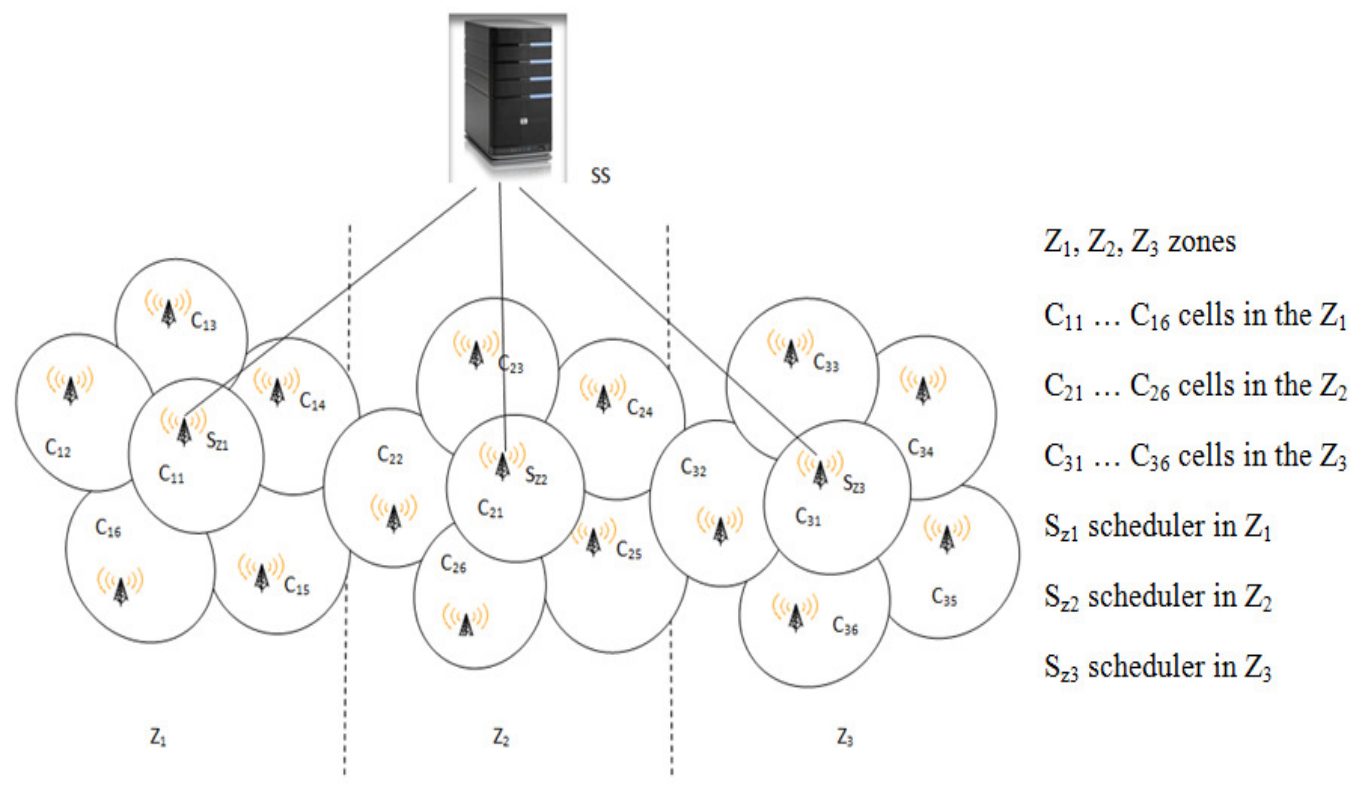

Figure 1. System model

The proposed mobile grid system model is shown in the figure 1. A mobile wireless network is considered with a cellular infrastructure composed of a wired backbone and a number of base stations (BSs). Each BS is in control of a cell, and a group of BSs forms a location area (LA). Since each LA consists of a number of cells, a zone partition concept is proposed to improve the granularity of location description. A zone is subset of an LA, which is composed of a group of adjacent cells. The service area of each zone consists of $n$ cells $(n=6)$ and it is controlled by a BS which at the centre of the zone. This BS is used as a gateway to the grid and it acts as the scheduler. It undertakes the role of the mediator between the mobile devices and the grid system, and tries to hide the instability of the wireless/mobile environment by acting on behalf of the mobile device. Mobile devices $(\mathrm{M})$ can be used as both resource consumers $\left(\mathrm{M}_{\mathrm{U}}\right)$ and resource providers $\left(\mathrm{M}_{\mathrm{RP}}\right)$.The $\mathrm{M}_{\mathrm{RP}}$ s provide the description of their capabilities and the degree of their availability to the scheduler. The scheduler is then responsible for decomposing incoming request and scheduling the overall execution by providing specific tasks to each of the participating $\mathrm{M}_{\mathrm{RP}} \mathrm{S}$. Scheduler can act as a mediator capable of hiding the heterogeneity of the $M_{R P}$ from the requesting node, coordinating the overall execution of the submitted job and allowing the $M_{R P}$ to appear to the rest of the network as an ordinary mobile resource. $A \mathrm{M}_{\mathrm{RP}}$ in a zone is expected to demonstrate the same, if not similar, movement behaviour. If a $\mathrm{M}_{\mathrm{RP}}$ is moving from one cell to another cell belonging to another zone, location registration and identity authorization need to be carried out. If scheduler cannot find a suitable $\mathrm{M}_{R P}$ in its zone, it passes the request to the Neighbouring Schedulers (NS) and if it does not find then it passes the request to the Super Scheduler (SS). The NS is the one which is closer to the current zone. The current scheduler has the details of its NSs. The SS has the entire information about the grid community.

Figure 2 illustrates the proposed mobile grid scheduler running on each BS. The components of the mobile grid scheduler are Resource Registry (RR), Availability Predictor (AP), Mapper, and Allocator. 


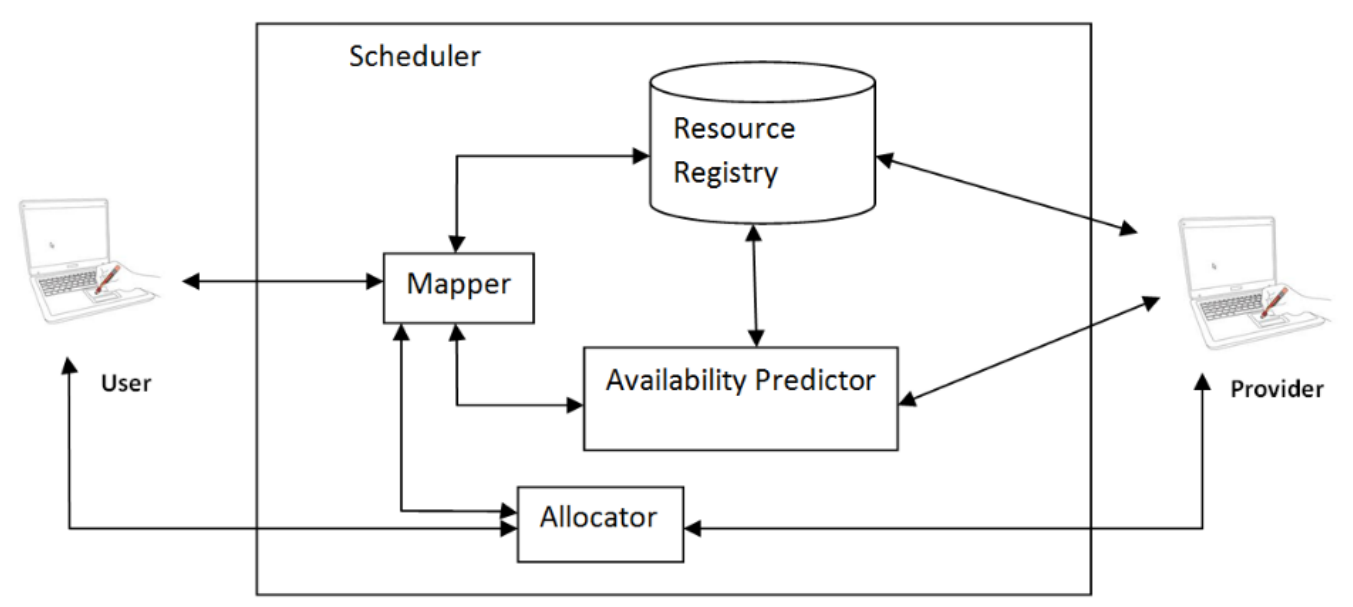

Figure 2. Proposed Mobile Grid Scheduler

\subsection{Resource Registry}

The information about the registered resources is stored in the resource registry in the following format.

$<$ resource id, system specification, movement pattern, battery info, zone info >

- resource id :

unique name for the particular resource

- system specification : processor and memory information

- movement pattern: unknown or known with total duration

- battery info: capacity and C-rate information

- zone info: no of cells and the scheduler information

\subsection{Availability Prediction}

The availability predictor is the one which predicts the dynamic availability of the registered $M_{R P}$.

\subsubsection{Physical availability prediction}

The physical availability is predicted based on movement type and movement model of the $\mathrm{M}_{\mathrm{RP}}$. The movement type helps to find the movement direction and the movement model prediction helps to locate the position of the $\mathrm{M}_{\mathrm{RP}}$ in near future.

The movement type is predicted based on the movement direction of $\mathrm{M}_{\mathrm{RP}}$ to the $\mathrm{BS}$. There are two movement types namely movement towards BS and movement apart from BS. The movement type is predicted based on the concept of Markov chain process i.e. the mobile resource current location only depends on the previous location of the mobile resource. The locations of the $M_{R P S}$ are found by the GPS which is assumed to be part of the $M_{R P}$. The distance between the $\mathrm{M}_{\mathrm{RP}}$ and the $\mathrm{BS}$ is found by using the Haversine formula. The previous distance to the $\mathrm{BS}\left(\mathrm{D}_{\beta}\right)$ is computed as,

$$
\mathrm{D}_{\beta}=\mathrm{R}^{*} \mathrm{c}
$$

where $\mathrm{R}$ is the radius of earth and $\mathrm{c}=2 * \operatorname{atan} 2(\sqrt{\mathrm{a}}, \sqrt{ }(1-\mathrm{a}))$

where $\mathrm{a}=\sin 2(\Delta \mathrm{lat} / 2)+\cos \left(\right.$ lat $\left._{\alpha}\right) \cos \left(\right.$ lat $\left._{\beta}\right) \sin 2(\Delta \mathrm{long} / 2)$ 
where $\Delta$ lat $=$ lat $_{\beta}-$ lat $_{\alpha}, \Delta$ long $=$ long $_{\beta}-$ long $\alpha, \alpha$ is location of BS and $\beta$ is previous location of $\mathrm{M}_{\mathrm{RP}}$

The current distance to the BS $\left(\mathrm{D}_{\lambda}\right)$ is derived from,

$$
\mathrm{D}_{\lambda}=\mathrm{R} * \mathrm{c}
$$

where $\mathrm{R}$ is the radius of earth and $\mathrm{c}=2 * \operatorname{atan} 2(\sqrt{\mathrm{a}}, \sqrt{ }(1-\mathrm{a}))$

where $\mathrm{a}=\sin 2(\Delta \mathrm{lat} / 2)+\cos \left(\right.$ lat $\left._{\alpha}\right) \cos \left(\right.$ lat $\left._{\lambda}\right) \sin 2(\Delta$ long $/ 2)$

where $\Delta$ lat $=$ lat $_{\lambda}-$ lat $_{\alpha}, \Delta$ long $=\operatorname{long}_{\lambda}-$ long $_{\alpha}, \alpha$ is location of BS and $\lambda$ is current location of $\mathrm{M}_{\mathrm{RP}}$

The $\mathrm{M}_{\mathrm{RP}}$ 's current direction is defined as the direction from its previous position to the current position. $\theta_{\mathrm{RP}}(\mathrm{t})$ is denoted as the moving direction of the $\mathrm{M}_{\mathrm{RP}}$, which is defined as the degree from the current direction clockwise or counter clockwise, i.e., $-\Pi<=\theta<=\Pi$. $\theta_{\mathrm{RP}}(\mathrm{t})$ is calculated based on Haversine formula. $\theta_{\mathrm{RP}}(\mathrm{t})$ is computed as

$$
\theta_{\mathrm{RP}}(\mathrm{t})=\operatorname{atan} 2\left(\sin (\Delta \mathrm{long}) \cdot \cos \left(\mathrm{lat}_{\lambda}\right), \cos \left(\mathrm{lat}_{\beta}\right) \cdot \sin \left(\mathrm{lat}_{\lambda}\right)-\sin \left(\mathrm{lat}_{\beta}\right) \cdot \cos \left(\mathrm{lat}_{\lambda}\right) \cdot \cos (\Delta \mathrm{long})\right)
$$

where $\Delta$ lat $=$ lat $_{\lambda}-$ lat $_{\beta}, \Delta$ long $=\operatorname{long}_{\lambda}-$ long $_{\beta}$

From (1) and (2), if the $D_{\beta}$ is greater than the $D_{\lambda}$ then the movement type is towards BS otherwise movement type is apart from the BS. The moving direction of the $\mathrm{M}_{\mathrm{RP}}$ computed by (3).

Movement of the $\mathrm{M}_{\mathrm{RP}}$ can be modelled as known and unknown models. The known movement model consists of identical movement patterns of each $\mathrm{M}_{\mathrm{RP}}$ which illustrates the distinguished behaviour of the movement of the $\mathrm{M}_{\mathrm{RP}}$ within a defined period of time. There are two types of known movement models namely movement circle (MC) and movement track (MT) models. The MC model is depended on the assumption that wherever a $M_{R P}$ moves from a location, the $M_{R P}$ will eventually return or has returned to its starting point. The MT is a unidirectional itinerary which begins and ends with a stationary location or a boundary location. A boundary location is a location at the boundary of the service area and a location is called a stationary location if the $\mathrm{M}_{\mathrm{RP}}$ has stayed in the location at least for a period of time. The physical availability of the known movement model can be computed as

$$
\mathrm{PA}=\mathrm{D}_{\mathrm{T}}-\mathrm{D}_{\mathrm{C}}
$$

where DT is the total life time of resource in a region and DC is the current duration of the $\mathrm{M}_{\mathrm{RP}}$ in the region.

The unknown movement model is the one whose movement pattern is random. The movement of a $\mathrm{M}_{\mathrm{RP}}$ can be modelled by a discrete parameter and discrete state stochastic process if and only if it is assumed that the movement is random. Therefore, the Markov chain model is used to describe the behaviour of the random parts of the user's movement. The Markov model assumes that location is random variable and the probability distributions for its future development depend only on the present location. The physical availability of the unknown movement model can be computed as

if $\mathrm{M}_{\mathrm{RP}}$ is in the LS cell then

$$
\text { PA }=\left(\mid\left(\text { BS Coverage Area }-D_{\lambda}\right) \mid+ \text { Boundary BS Coverage Area }\right) / \text { Mobility }
$$

else

$$
\mathrm{PA}=\left(\mathrm{BS} \text { Coverage Area }-\mathrm{D}_{\lambda}\right) / \text { Mobility }
$$

where Mobility is the time taken by the $\mathrm{M}_{\mathrm{RP}}$ to move from one location to another location and determined by

$$
\text { Mobility }=\left(D_{\beta}-D_{\lambda}\right) / \text { Time Taken to Reach from } \beta \text { to } \lambda
$$




\subsubsection{Battery power prediction}

The battery availability is computed based on the battery capacity $(\mathrm{C}), \mathrm{C}$ - rate and the current battery power usage. A battery's capacity is measured in Amp-hours, called " $\mathrm{C}$ " is the theoretical amount of current a battery drops when discharged in one hour to the point of $100 \%$ depth of discharge. Battery manufacturers determine the capacity by different methods. However the faster the discharge, that lowers the capacity of the battery. The charge and discharge current of a battery is measured in C-rate. Most portable batteries, with the exception of the lead acid, are rated at $1 \mathrm{C}$. A discharge of $1 \mathrm{C}$ draws a current equal to the rated capacity. The capacity of a battery is commonly measured with a battery analyzer. If the analyzer's capacity readout is displayed in percentage of the nominal rating, 100 percent is shown if $1000 \mathrm{~mA}$ can be drawn for one hour from a battery that is rated at $1000 \mathrm{mAh}$. The discrepancy in capacity readings with different C-rates largely depends on the internal resistance of the battery. On a new battery with a good load current characteristic or low internal resistance, the difference in the readings is only a few percentage points. On a battery exhibiting high internal resistance, the difference in capacity readings could swing plus/minus 10 percent or more. To compensate for the different readings at various discharge currents, error factor is included in battery availability prediction. The error factor is calculated based on the users CPU requirement. Applying the error factor does not improve battery performance; it merely adjusts the availability calculation if discharged at a higher or lower C-rate than specified.

The available battery duration $\left(\mathrm{B}_{\mathrm{AD}}\right)$ is determined by

$$
\mathrm{B}_{\mathrm{AD}}=\left(\mathrm{B}_{\mathrm{TD}}-\mathrm{B}_{\mathrm{CD}}\right) \pm \text { Error Factor }
$$

where the $\mathrm{B}_{\mathrm{TD}}$ is the total battery duration and it is determined by

$$
\mathrm{B}_{\mathrm{TD}}=\mathrm{C} / \mathrm{C}-\text { rate }
$$

and $\mathrm{B}_{\mathrm{CD}}$ is the current battery duration and it is determined by

$$
\mathrm{B}_{\mathrm{CD}}=\text { current Battery Power in percentage } * \mathrm{~B}_{\mathrm{TD}}
$$

\subsection{Mapper}

The mapper performs following mapping process namely user identification, system specification and task requirement mapping. The user identification is done by mapping the user id in the RR, the system specification mapping is done by checking the system specification in the RR, and task requirement mapping is done by mapping the task requirement with the dynamically predicted resource availability. If the user requirements are met then these details about the resources are sent to the resource allocator.

\subsection{Allocator}

The allocator checks priority of the resource to ascertain whether the task can execute on the available resources and meet the user-specified deadline. The resource allocator sets priority to registered resource based on availability factor (Physical Availability Factor (PAF) and Battery Availability Factor (BAF)) of the resource. If the availability of the resource is high, then the resource gets more priority than the other resources.

The PAF is calculated based on the physical availability and zone residence time (calculated based on the current velocity of the resource provider).

$$
\text { PAF }=\text { Physical Availability /Zone Residence Time }
$$

The MRP's physical availability is full if the PAF is more than 0.60 (priority will be full). When the PAF is 0.30 to 0.60 then the physical availability is high (priority will be high) and the physical availability is low (priority will be low) when PAF is less than 0.30. 
The BAF is calculated based on the battery availability and total battery power (based on the current battery usage).

$$
\mathrm{BAF}=\text { Battery Availability / Total Battery Availability }
$$

The battery availability is full if the BAF is more than 0.60 (priority will be full). When the BAF is 0.30 to 0.60 then the battery availability is high (priority will be medium) and the battery availability is low (priority will be low) when BAF is less than 0.30 .

\section{Proposed Algorithm}

In this section, a scheduling algorithm is presented, which incorporates mobility and battery power. Since the mobile grid resources fluctuate over time, the task requirements are checked dynamically during application runtime. In an attempt to efficiently deal with the dynamism of mobile grid resources, the algorithm adopts a resource prediction technique that is mainly helpful in avoiding serious scheduling problems. The scheduler predicts the resource information based on mobility and battery power which is maintainable by the scheduler while scheduling the tasks. However, it is not assumed that the information is available for the next invocation of the application. When a $M_{R P}$ or $M_{U}$ enters into a zone it register itself in the local scheduler by sending its id $\left(M_{R P}\right.$ id or $M_{U}$ id), and the scheduler will authenticate $M_{R P}$ or $M_{U}$ by checking its id in the SS. Table 1 gives the message format used by the resource as well as the consumer and Table 2 gives the different types of flags used to differentiate data from control information. The proposed algorithm is given in the figure 3.

Table 1. Message format

\begin{tabular}{|c|c|c|c|}
\hline 2 bytes & 2 bytes & 2bits & 6bytes \\
\hline SRC & DEST & FLAG & Data/ACK \\
\hline
\end{tabular}

Table 2. Flag

\begin{tabular}{|c|c|}
\hline FLAG & MEANING \\
\hline 00 & DATA \\
\hline 01 & ACK \\
\hline 10 & NACK \\
\hline 11 & SEND \\
\hline
\end{tabular}

\section{SimULATION ENVIRONMENT}

Scheduling algorithm is evaluated using SimGrid toolkit [9] because of its rich set of simulation facilities that easily enables the development and evaluation of scheduling algorithms for heterogeneous distributed computing environments (for example, grids).A mobile grid environment is simulated with three zones, each zone has six cells (2000 meters 2000 meters) and each cell has five mobile resources (at the start of simulation). It is assumed that the tasks are independent. The scheduler knows the internal task arrival rates and available 
International Journal of Grid Computing \& Applications (IJGCA) Vol.3, No.2, June 2012

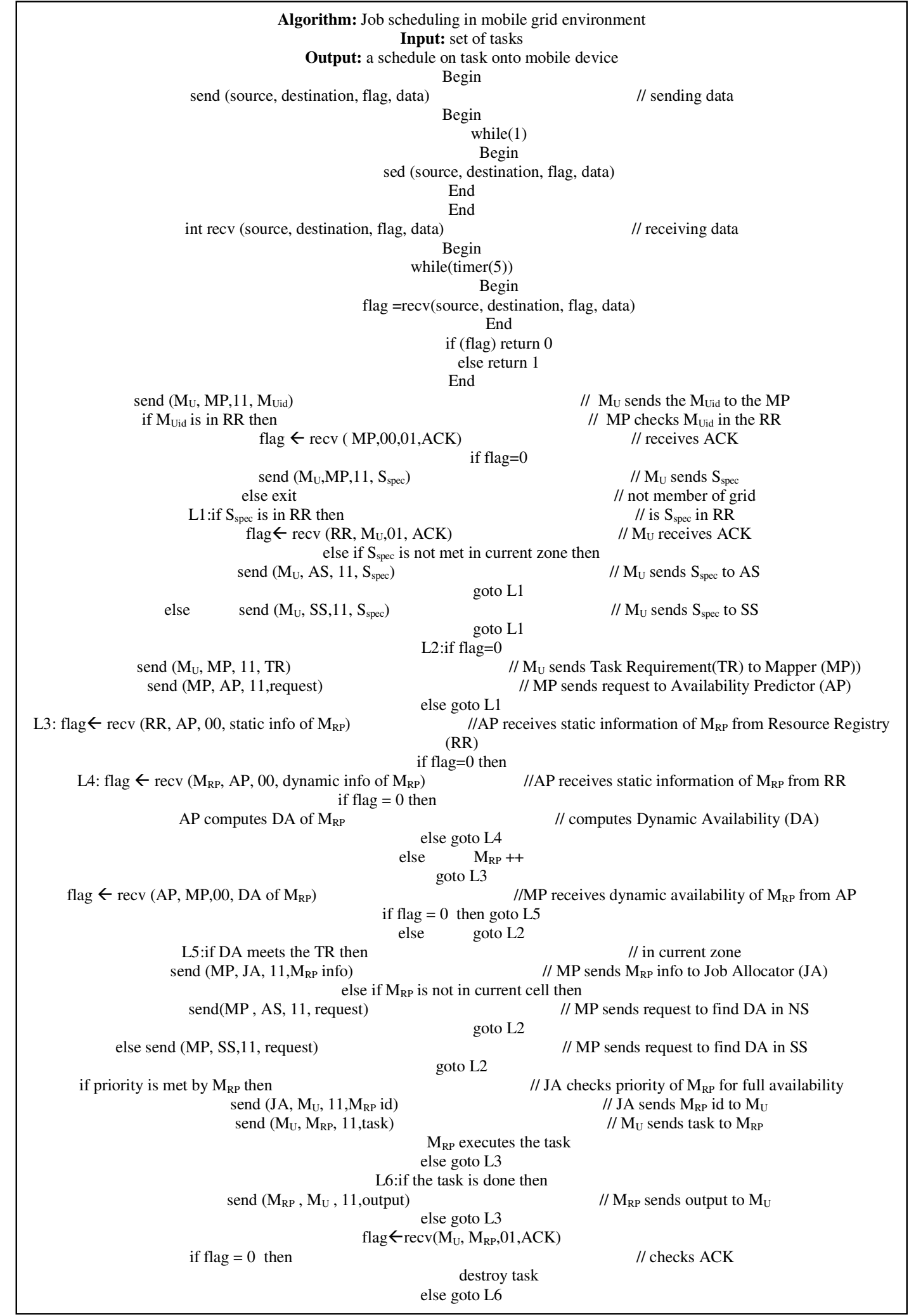

Figure 3. Proposed algorithm 
bandwidth for all the resources. The immediate mode input model is used for the task distribution. The tasks are considered with varying execution time. The available bandwidth is fixed and is equally distributed among the resources. It is assumed that once the resource is allotted for a task, it is available till the end of the task completion. There are number of parameters associated with mobile devices such as mobility, battery, CPU and memory, and in this the proposed work considers mobility and battery power for simulation. Each mobile device in the simulated environment has a maximal radio range of $2000 \mathrm{~m}$, and moves based on the known and unknown mobility model. The speed of each mobile node is from 0 to $10 \mathrm{~m} / \mathrm{s}$. The speeds of mobile devices have wide scope: some nodes are pedestrians, some nodes are vehicles. Mobile devices dynamically enter and leave the mobile grid. The initial values of mobile device's battery capacity $([1.2,2.2,3.4,4.4] \mathrm{KWPH}$ and the current values are also initialized with random values and changes dynamically with random values, for battery $[0,1, \ldots, 5]$. Figure 5 shows the mobility pattern and battery power of ten mobile resources.

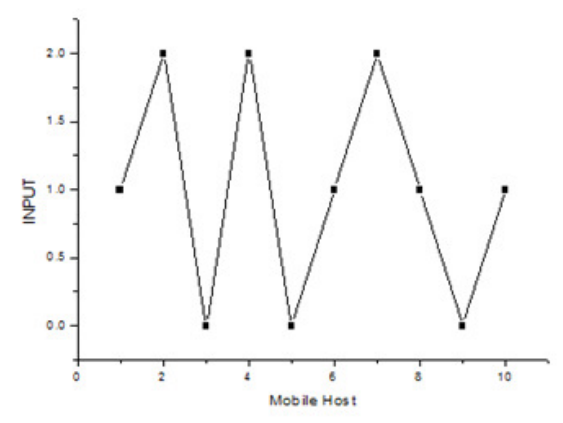

A

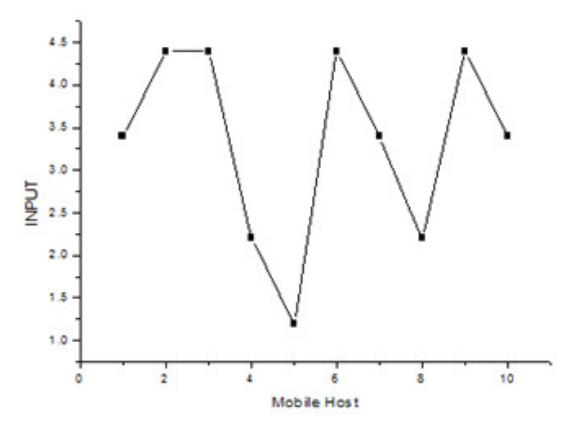

B

Figure 4. A. mobility pattern B. battery power

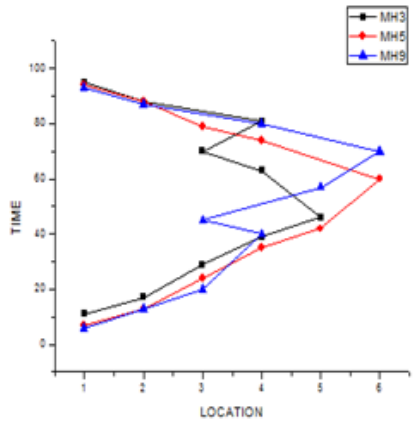

A

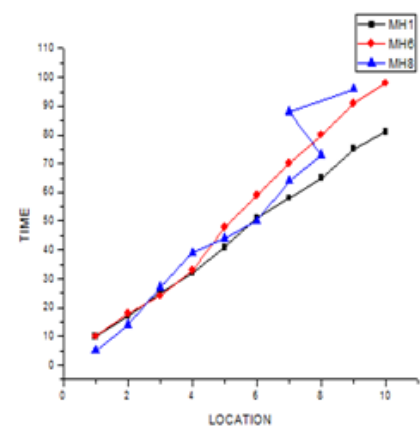

B

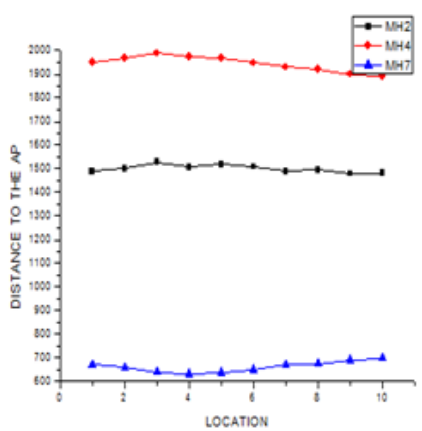

$\mathrm{C}$

Figure 5. Movement pattern A. Movement circle B. Movement track C. Random

\subsection{Experimental results}

The experiments were conducted using investigated effects of two factors (i.e. resource physical availability and battery power) on execution time.

Figure 6 shows the effect of resource's physical availability and battery power on execution time when different number of jobs is executed with single cell environment. The effect of resource 
physical availability and battery power is experimented for various PAF and BAF. As shown in the figure 6 , when the PAF $>0.60$ and $\mathrm{BAF}>0.60$ reports the shortest execution time this is due the higher priority of mobile resources. When $\mathrm{PAF}$ is $0.30<\mathrm{PAF}<=0.60$ and $\mathrm{BAF}$ is $0.30<\mathrm{BAF}<=$ 0.60 then the scheduler searchers for the other mobile resources with high priority within the cell and if it finds then sends the tasks to that resource. If it does not find any other resources then it checks the user requirement and if it is met then sends the job to that mobile resource. When the $\mathrm{PAF}$ and $\mathrm{BAF}$ are low the scheduler searches for higher priority mobile resources both within the cell and within other cells. If it finds the mobile devices with higher priority, then the scheduler sends the job to that mobile device. Otherwise it checks user requirement and sends the job to that resource. If the requirement is not met then the scheduler sends the job to the SS.
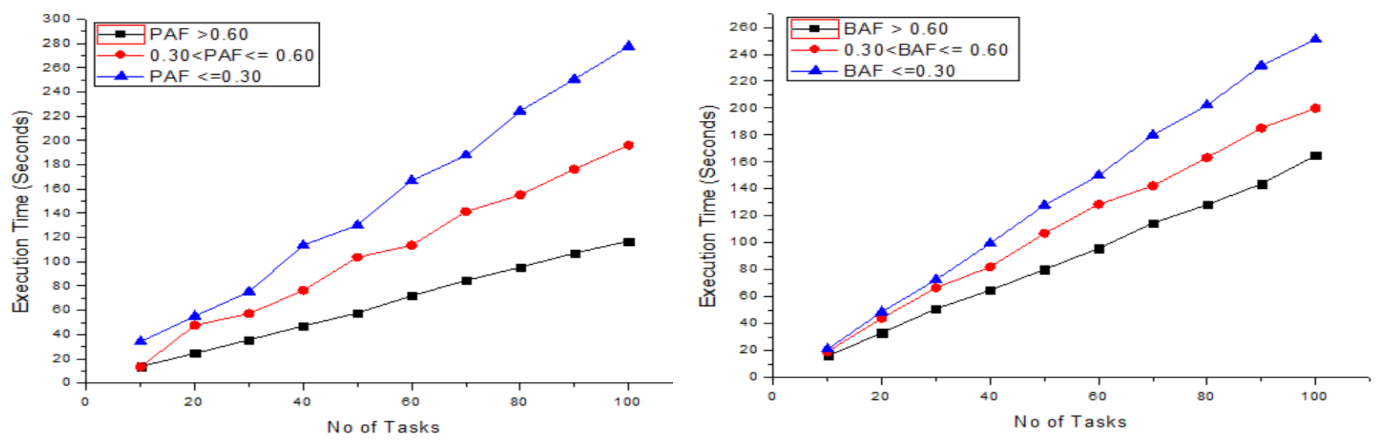

Figure 6. Effect physical availability and battery availability on execution time in single cell environment

A separate set of comparisons, in similar simulation settings has been performed to evaluate the performance of proposed work. The figures 7, 8, 9 and 10 show the effect of physical availability and battery availability on execution time for uniform task size and varying task size with different number of cells in the zone. The effect of resource physical availability and battery power is experimented using PAF and BAF. In figures 7 and 9, as the number of cells in the zone increases the resource residence time in the zone increases which in turn increase the physical availability of resource. As the number of resources with increased physical availability increases then the resource prediction time decreases which in turn reduces the execution time of the task.
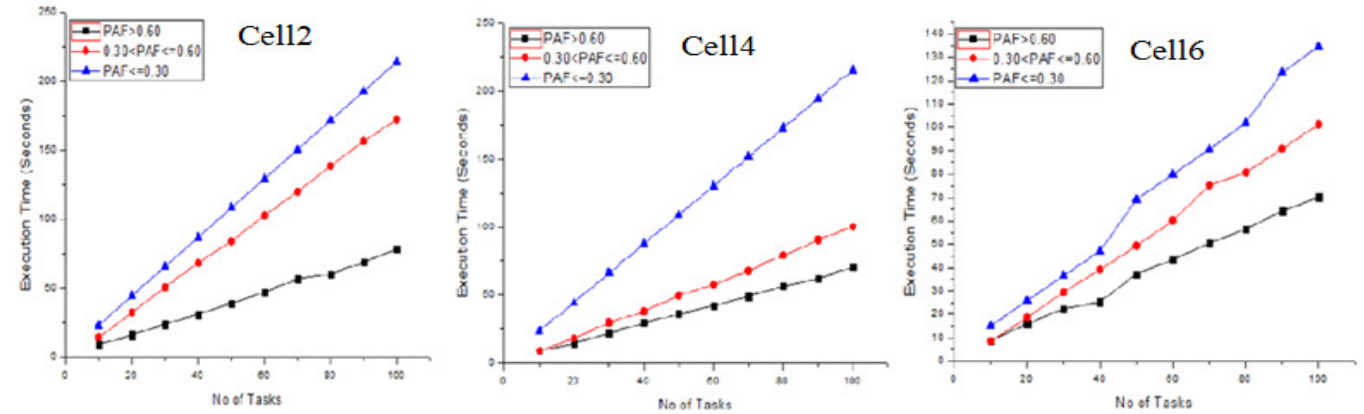

Figure 7. Effect of physical availability on execution time for uniform task size in zone environment

As shown in the figures, when the PAF $>0.60$ and $\mathrm{BAF}>0.60$ reports the shortest execution time this is due to the higher priority of mobile resources. When the resource has the higher priority the resource prediction time is reduced which in turn reduces the total execution time of the task. 
International Journal of Grid Computing \& Applications (IJGCA) Vol.3, No.2, June 2012

In the proposed work the resource physical availability is increased due to the increase in the resource residence time in the zone. When $\mathrm{PAF}$ is $0.30<\mathrm{PAF}<=0.60$ and $\mathrm{BAF}$ is $0.30<\mathrm{BAF}<=$ 0.60 then the scheduler searchers for the other mobile resources with high priority within the zone and if it finds then sends the tasks to that resource. If it does not find any other resources then it checks the user requirement and if it is met then sends the job to that mobile resource otherwise it sends the task to the NS. When the PAF and BAF are low the scheduler searches for higher priority mobile resources both within the zone and within other zones. If it finds the mobile devices with higher priority, then the scheduler sends the job to that mobile device. Otherwise it checks user requirement and sends the job to that resource. If the requirement is not met then the scheduler sends the job to the SS.
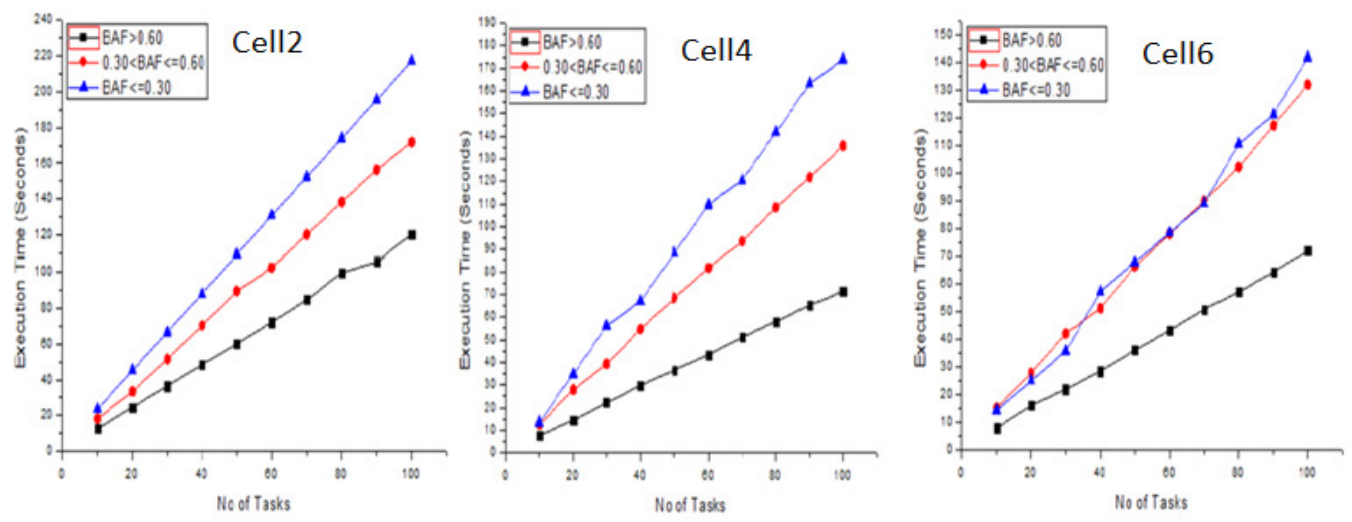

Figure 8. Effect of battery power on the execution time for uniform task size in zone environment
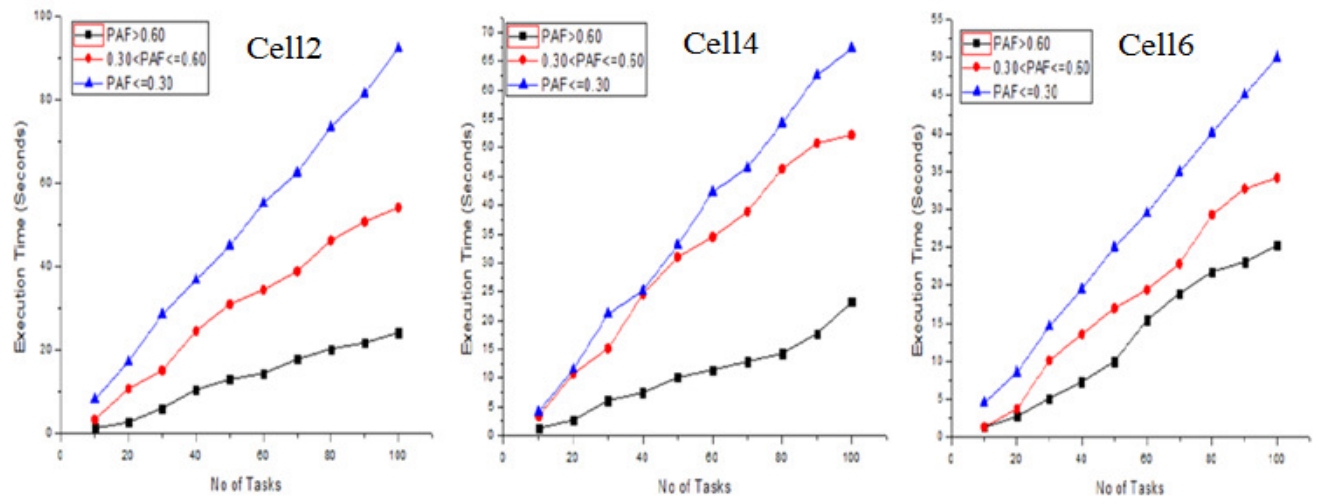

Figure 9. Effect of physical availability on execution time for varying task size in zone environment 
International Journal of Grid Computing \& Applications (IJGCA) Vol.3, No.2, June 2012
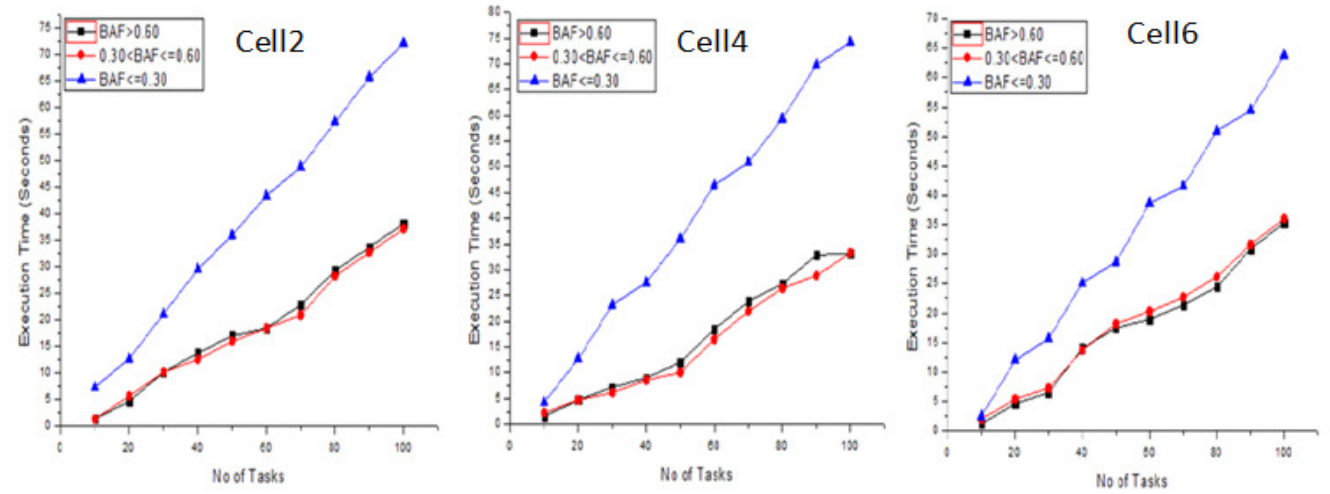

Figure 10. Effect of battery power on the execution time for varying task size in zone environment

\section{CONCLuSiON}

This paper has presented a scheduling algorithm, for predicting resource availability and allocating the independent and varying execution time tasks to the mobile resources in mobile grid environments. They are carefully designed to incorporate the fundamental characteristics of the mobile grid (that is, dynamism and heterogeneity) into the decision-making process. The physical availability prediction uses the movement type, movement pattern and movement direction of the resource providers. The experiment results have shown the effect of physical availability and battery availability on execution time both in single cell and zone environment. The simulation results have presented in this paper clearly show the promising improvement of the proposed work compared to the previous work. The proposed work can be extended to include other dynamic parameters of mobile resources such as CPU and memory to increase the performance of the proposed system.

\section{REFERENCES}

[1] A. M. Otebolaku, J. S. Iyilade, \& M. O. Adigun, (2008) "CAAM: A context aware adaptation model for mobile grid service infrastructure", Proceedings of the 11th IEEE International Conference on Computational Science and Engineering, pp. 419-425.

[2] N. Roy, \& S. K. Das, (2009) "Enhancing Availability of Grid Computational Services to Ubiquitous Computing Applications", IEEE Transactions on Parallel and Distributed Systems 20(7), 953-967.

[3] S. Stephen Vaithiya \& S Mary Saira Bhanu, (2011) "Mobility and Battery Power Prediction based Job Scheduling in Mobile Grid Environment", Proceedings of the International Conference on Parallel, Distributed Computing technologies and Applications (PDCTA 2011), CCIS 203, CSpringer-Verlag Berlin Heidelberg, pp. 312-322.

[4] Venkata Durga Kiran. Kasula, (2007) "Performance Analysis of Layered Architecture to Integrate Mobile Devices and Grid Computing with a Resource Scheduling Algorithm", Proceedings of the International Conference on Computational Intelligence and Multimedia Applications, vol. 4, pp. 352-356.

[5] G. Preetam, R. Nirmalya, S. Das \& K. Basu, (2005) "A Pricing Strategy for Job Allocation in Mobile Grids using a Non-Cooperative Bargaining Theory Framework", Special Issue on Design and Performance of Networks for Super-Cluster and Grid-Computing, pp. 1366-1383.

[6] C.-Q. Huang, Z.-T. Zhu, Y.-H. Wu \& Z.-H. Xiao, (2006) "Power-Aware Hierarchical Scheduling with Respect to Resource Intermittence in Wireless Grids", Proceedings of the Fifth International Conference on Machine Learning and Cybernetics, Dalian, pp. 693-698. 
International Journal of Grid Computing \& Applications (IJGCA) Vol.3, No.2, June 2012

[7] U. Farooq \& W. Khalil, (2006) "A Generic Mobility Model for Resource Prediction in Mobile Grids", Proceedings of the International Symposium on Collaborative Technologies and Systems.

[8] J. Lee, H. Lee, K. Chung, \& H. Yu, (2009) "Balanced Scheduling Algorithm Considering Availability in Mobile Grid", Springer, Heidelberg, pp. 212-222.

[9] H. Casanova, (2001) "Simgrid: A Toolkit for the Simulation of Application Scheduling", Proceedings of 1st IE IEEE/ACM International Symposium on Cluster Computing and the Grid.

\section{Authors}

Stephen Vaithiya $\mathbf{S}$ received the B.E Degree in Electrical and Electronic Engineering from Anna University, Chennai in 2007 and the M.E Degree i Software Engineering from Anna University, Tiruchirappalli in 2009. Currently, $\mathrm{r}$ is pursuing the Ph.D. Degree in the Department of Computer Science an Engineering in National Institute of Technology, Tiruchirappalli, India. His researc interests include Mobile, Grid and Cloud Computing.

Mary Saira Bhanu S received the B.E Degree in Electronics and communicatior from Madurai Kamaraj University in 1986, the M.E Degree in Computer Science from Bharathidasan University in 1989 and the Ph.D. Degree from the Departmen of Computer Science and Engineering from National Institute of Technology Tiruchirappalli in 2009.Currently, she is a Associate Professor in the Department o. Computer Science and Engineering in National Institute of Technology Tiruchirappalli, India. Her research interests include OS, Real Time Systems

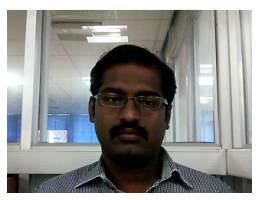
Distributed Computing, Grid Computing and Cloud Computing.

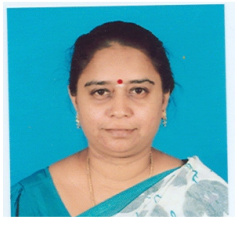

